\section{Algorithm for Aging Viscoelastic Structures Under Periodic Load}

\section{By Zdeněk P. Bažant, ${ }^{1}$ F. ASCE and Tong-Sheng Wang ${ }^{2}$}

Anstract: A numerical step-by-step algorithm for the analysis of concrete structures exposed to a periodic history of environmental humidity or temperature is presented. The creep law of concrete is assumed to be linear, and the relationship between humidity and shrinkage is also linear. Cracking is assumed to be absent. The effect of concrete age on creep properties is taken into account. The creep law is considered in a rate-type form corresponding to the complex variables to describe the periodic part of the response. generalized to cannot be separated in adribe the periodic part of the response. Since this part cannot be separated in advance from the drifting mean response, the standard exponential algorithm in real variables and the new one in complex variables are used simultaneously in each time step to provide the total response. The algorithm allows an arbitrary increase of the time step, and time steps that are orders of magnitude larger than the fluctuation period, as well as the relaxation times, are possible without causing inaccuracies and numerical instability. The algorithm leads to a series of incremental elastic problems in which the stresses, spatial problems are solved by finite ful for spectral aralysis of the response of concrete proposed algorithm is usedom environmental humidity response of concrete structures exposed to rancomputation time hum when high frequencies are present in the spectral density of environment.

\section{INTRODUCTION}

In certain structural analysis problems, the analyst needs to solve the history of stresses, strains, and deformations in an aging viscoelastic structure subjected to a periodic loading. This is the case, for example, in predictions of long-term response of concrete structures to a randomly fluctuating environmental humidity or temperature of a given spectral density distribution. The response can, in principle, be obtained by the impulse response function method, however, this approach is effective only for those few problems that can be solved analytically (21). Normally, the spatial solution has to be obtained by finite elements, and as long as the problem can be treated as linear, the spectral approach is then computationally much more efficient than the impulse response function approach. There are two reasons for this: (1) The spectral densities of input and response are related algebraically while the autocorrelation functions of input and response are related by integrals; and (2) the environment can be adequately described by only a few sinusoidal components.

The spectral method is normally formulated for nonaging linear systems $(13-15,18,19)$. Due to the very strong effect of the age of concrete

${ }^{1}$ Prof. of Civ. Engrg. and Dir., Center for Concrete and Geomaterials, The Technological Inst., Northwestern Univ., Evanston, IIl. 60201.

${ }^{2}$ Visiting Scholar, Northwestern Univ.; on leave from the Huai River Commission, Bangbu, Anhui, China.

Note.-Discussion open until November 1, 1984. To extend the closing date one month, a written request must be filed with the ASCE Manager of Technical and Professional Publications. The manuscript for this paper was submitted for review and possible publication on October 27, 1983. This paper is part of the Joumal of Engineering Mechanics, Vol. 110, No. 6, June, 1984. OASCE, ISSN 0733-9399/84/0006-0972/\$01.00. Paper No. 18911 . on its creep, the spectral method needs to be generalized for aging systems, which was recently done in Refs. 6-7. The variance, and all other statistical characteristics of the random process of stress or deformation at any point of the structures, can then easily be obtained if one solves the frequency response function, $H\left(\omega, x, t, t_{0}\right)$, which describes the response at age $t$ and location $x$ to a periodic (sinusoidal) loading (e.g., surface humidity variation) of any single frequency, $\omega\left(t_{0}\right.$ is the age at which the structure is exposed to this loading). For some problems, such as the shrinkage stresses in an aging viscoelastic half-space, the frequency response function can be obtained by numerical evaluation of certain integrals (9). For arbitrary structures, a solution of the frequency response function by the finite element method, in which the stresses, strains, and displacements are treated as complex variables, was recently developed (10). In this formulation, the frequency response function is obtained by step-by-step numerical integration in time. However, the time step must be kept smaller than about $1 / 16$ of the period $2 \pi / \omega$, which leads to a prohibitively large number of time steps and a large cost of computation if the prescribed loading spectrum involves components whose time period is short (less than $1 \mathrm{yr}$ ).

For aging creep of concrete structures under steady loadings, a tremendous reduction of computation time is achieved by the well-known exponential algorithm $(3-5,11)$ for a rate-type form of the aging creep law, which represents an extension of a previous algorithm for nonaging creep $(20,24)$. The purpose of this paper is to generalize this algorithm to periodic loads superimposed on a slowly drifting mean which drifts at a decaying rate. As will be shown, this generalization will allow the time step to be increased in a geometric progression and reach values that are orders of magnitude larger than the period of the loading without any significant loss of accuracy.

\section{Constitutive Relation}

For many practical purposes, the creep law of concrete may be assumed to be linear and obey the principle of superposition (for conditions of validity see Refs. 5, 16, 17). Taking into account the isotropy of the material, we may then write the creep law in the form

$\epsilon(t)=\int_{t_{0}}^{t} J^{V}\left(t, t^{\prime}\right) d \sigma\left(t^{\prime}\right)+\epsilon^{0}(t), \quad \epsilon_{i j}^{D}(t)=\int_{t_{0}}^{t} J^{D}\left(t, t^{\prime}\right) d \sigma_{i j}^{D}\left(t^{\prime}\right) \ldots \ldots \ldots(1)$ (1)

in which $\epsilon^{0}(t)=$ the given shrinkage or thermal strain at age $t ; \epsilon, \epsilon_{i j}^{D}=$ volumetric and deviatoric parts of strain tensor $\epsilon_{i j}$ in cartesian coordinates $x_{i}(i=1,2,3)$, i.e., $\epsilon_{i j}=\epsilon_{i j}^{D}+\delta_{i j} \epsilon ; \delta_{i j}=$ Kronecker delta; $\sigma_{,} \sigma_{i j}^{D}=$ volumetric and deviatoric parts of the total stress tensor $\sigma_{i j}$; and $J^{v}\left(t, t^{\prime}\right)$, $I^{D}\left(t, t^{\prime}\right)=$ volumetric and deviatoric compliance functions representing the volumetric and deviatoric strains at age $t$ caused by a unit volumetric or deviatoric stress acting since age $t^{\prime}$. These functions, which fully characterize creep and elastic behavior, may be expressed easily in terms of the uniaxial compliance function $J\left(t, t^{\prime}\right),(5)$. The aging of the material, a very important trait of concrete creep, is described by the fact that the compliance functions depend separately on $t$ and $t^{\prime}$ rather than only on the time lag $t-t^{\prime}$. 
For realistic forms of the compliance function, structural analysis has to be carried out numerically in time steps. As one possibility, this may be based on approximating the integrals in Eq. 1 by finite sums. However, the resulting algorithm is prohibitively inefficient for systems with more than a few unknowns $(5,12)$. A vastly more efficient numerical algorithm may be obtained if the integral-type creep law in Eq. 1 is approximated by a rate-type creep law which can be done with any desired accuracy. The best form of the rate-type creep law is the one based on the Maxwell chain model which reads as

$\boldsymbol{\sigma}=\sum_{\mu=1}^{m} \sigma_{\mu}, \quad \sigma_{i j}^{D}=\sum_{\mu=1}^{m} \sigma_{i j_{\mu}}^{D}$

$\dot{\boldsymbol{\epsilon}}-\dot{\boldsymbol{\epsilon}}_{0}=\frac{\dot{\sigma}_{\mu}}{3 K_{\mu}(t)}+\frac{\sigma_{\mu}}{3 \eta_{\mu}(t)}, \quad \dot{\epsilon}_{i j}^{D}=\frac{\dot{\sigma}_{i j}^{D}}{2 G_{\mu}(t)}+\frac{\sigma_{i j}^{D}}{2 \eta_{\mu}^{D}(t)}$.

in which $\sigma_{\mu}=$ the partial stresses (internal variables) corresponding to the individual units of the Maxwell chain model $(\mu=1,2, \ldots m) ; K_{\mu}(t)$ and $G_{\mu}(t)=$ the elastic bulk and shear moduli associated with the $\mu$ th unit; and $\eta_{\mu}(t), \eta_{\mu}^{D}(t)=$ the corresponding bulk and shear viscosities at age $t$. Superimposed dots denote time rates. Due to isotropy, $K_{\mu}(t)=$ $E_{\mu}(t) /(3-6 v), G_{\mu}(t)=E_{\mu}(t) /(2+2 v)$, in which $E_{\mu}(t)$ is the elastic modulus for the $\mu$ th unit at age $t$, and $\nu$ is the Poisson ratio which may be considered for concrete as time-independent and equal to its elastic value. The viscosities $\eta_{\mu}(t)$ and $\eta_{\mu}^{D}(t)$ depend on temperature $T(5)$. For reference temperature $T=T_{0}\left(=25^{\circ} \mathrm{C}\right)$, one writes $\eta_{\mu}(t)=\tau_{\mu} K_{\mu}(t), \eta_{\mu}^{D}(t)$ $=\tau_{\mu} G_{\mu}(t)$ in which $\tau_{\mu}$ are the relaxation times of the Maxwell chain model. The $\tau_{\mu}$-values cannot be determined from the given creep data but must be chosen in advance in an appropriate manner. They must be spaced uniformly in the logarithmic time scale and must cover the entire time range of interest. Spacing by decades, i.e., $\tau_{\mu}=10^{\mu-1} \tau_{1}(\mu$ $=1,2, \ldots m-1$ ) is sufficient. The last value may be set very large, e.g., $\tau_{m}=10^{30}$, which makes the last unit of the chain equivalent to a spring.

For structures that are exposed, beginning with a certain age $t_{0}$, to a periodic environment, the histories of stress and strain may be expected to consist of fluctuations about a drifting mean, and, thus, may be described by the complex-valued stresses and strains

$\sigma_{i j}(t)=\hat{\sigma}_{i j}(t)+\tilde{\boldsymbol{\sigma}}_{i j}(t) e^{i \omega t}, \quad \boldsymbol{\epsilon}_{i j}(t)=\hat{\boldsymbol{\epsilon}}_{i j}(t)+\tilde{\boldsymbol{\epsilon}}_{i j}(t) e^{i \omega t}$

The actual stresses and strains are the real parts of these variables; $\hat{\sigma}_{i j}(t)$ and $\hat{\epsilon}_{i j}(t)$ represent the mean stresses and strains (real variables); $\tilde{\sigma}_{i j}(t)$ and $\bar{\epsilon}_{i j}(t)$ represent the complex-valued amplitudes of stresses and strains $i=$ imaginary unit; and $\omega=$ circular frequency of the periodic environment. The shrinkage (or thermal) strains may be considered in a similar form:

$\boldsymbol{\epsilon}^{0}(t)=\hat{\boldsymbol{\epsilon}}^{0}(t)+\tilde{\boldsymbol{\epsilon}}^{0}(t) e^{i \omega t}$

\section{Review of Algorithm for Steady Loading}

The mean components, denoted by superimposed hats, vary at a rate decaying roughly as $1 / t$, same as for the case of steady loads. For this case the most efficient solution is given by the so-called exponential algorithm known since 1971 (3). In contrast to the usual step-by-step methods for first-order ordinary differential equations, this algorithm permits a gradual increase of the time step without causing numerical instability or impairing accuracy. This is so even if the time step becomes orders of magnitude larger than the first relaxation time, $\tau_{1}$. The algorithm (3-5) leads to the following quasi-elastic incremental stress-strain relation:

$\Delta \hat{\boldsymbol{\sigma}}_{r}=3 K^{\prime \prime} \Delta \hat{\epsilon}_{r}-\Delta \sigma^{\prime \prime}, \quad \Delta \hat{\sigma}_{i j_{r}}^{D}=2 G^{\prime \prime} \Delta \hat{\epsilon}_{i j_{r}}^{D}-\Delta \sigma_{i j}^{D^{\prime \prime}}$.

in which $K^{\prime \prime}=\sum_{\mu=1}^{n} \lambda_{\mu} K_{\mu}\left(t_{r+1 / 2}\right), \quad G^{\prime \prime}=\sum_{\mu=1}^{n} \lambda_{\mu}^{\prime} G_{\mu}\left(t_{r+1 / 2}\right) \ldots \ldots \ldots \ldots$

$\Delta \sigma^{\prime \prime}=\sum_{\mu=1}^{m} \sigma_{\mu,}\left(1-e^{-\Delta z_{\mu}}\right)+3 K^{\prime \prime} \Delta \hat{\epsilon}^{0}$

$\Delta \sigma_{i j}^{D^{\mu \prime}}=\sum_{\mu=1}^{m} \sigma_{i j_{\mu, r}}^{D}\left(1-e^{-\Delta z_{\mu}^{\prime}}\right)$

$\Delta z_{\mu}=\frac{\Delta t}{\tau_{\mu}}, \quad \Delta z_{\mu}^{\prime}=\frac{\Delta t}{\tau_{\mu}^{\prime}}, \quad \lambda_{\mu}=\frac{1-e^{-\Delta z_{\mu}}}{\Delta z_{\mu}}$,

$\lambda_{\mu}^{\prime}=\frac{1-e^{-\Delta z_{\mu}^{\prime}}}{\Delta z_{\mu}^{\prime}}, \quad \tau_{\mu}=\frac{\bar{\eta}_{\mu}}{\bar{K}_{\mu}}, \quad \tau_{\mu}^{\prime}=\frac{\bar{\eta}_{\mu}^{D}}{\bar{G}_{\mu}}$.

Here, $\Delta$ denotes the time increments during the time step $\Delta t=t_{r+1}-$ $t_{r}$; and $r=1,2,3 \ldots$ are the discrete times, best chosen so that their spacing in the scale of $\log \left(t-t^{\prime}\right)$ would be uniform. Four, and for cruder results even two, steps per decade usually suffice. $\Delta \sigma^{\prime \prime}$ and $\Delta \sigma_{i j}^{D^{n}}$ are the inelastic stress increments which are taken into account in finite element analysis by inelastic nodal force increments obtained, according to the principle of virtual work, by integration over the element volume. Since the stress-strain relation in Eq. 6 is isotropic, the changes of displacements, strains, and stresses during $\Delta t$ may be solved by standard finite element analysis in which $K^{\prime \prime}$ and $G^{\prime \prime}$ are used as the elastic moduli (they are different in each time step). The values labeled by superimposed bars represent the values for the middle of the time interval in the log-time scale, i.e., for the time $t_{r+1 / 2}=t_{1}+\left[\left(t_{r}-t_{1}\right)\left(t_{r+1}-t_{1}\right)\right]^{1 / 2}$; and

$\bar{K}_{\mu}=K_{\mu}\left(t_{r+1 / 2}\right), \quad \bar{G}_{\mu}=G_{\mu}\left(t_{r+1 / 2}\right)$,

$\bar{\eta}_{\mu}=\eta_{\mu}\left(t_{r+1 / 2}\right), \quad \bar{\eta}^{D}=\eta_{\mu}^{D}\left(t_{r+1 / 2}\right)$

After solving by finite elements the incremental elastic problem, the values of partial stresses for the next discrete time may be obtained from the equations

$\hat{\boldsymbol{\sigma}}_{\mu, r+1}=\hat{\boldsymbol{\sigma}}_{\mu, r} e^{-\Delta x_{\mu}}+3 \bar{K}_{\mu} \lambda_{\mu}\left(\Delta \hat{\boldsymbol{\epsilon}}-\Delta \hat{\boldsymbol{\epsilon}}^{0}\right)$,

$\hat{\boldsymbol{\sigma}}_{i j_{\mu, r+1}}^{D}=\hat{\boldsymbol{\sigma}}_{i j_{\mu, r}}^{D} e^{-\Delta z_{\mu}^{\prime}}+2 \bar{G}_{\mu} \lambda_{\mu}^{\prime} \Delta \tilde{\epsilon}_{i j}^{D} \cdots$ 


\section{Derivation of Recurrent Formulas for Fluctuating Components}

We now consider the fluctuating components alone, i.e.

$\sigma_{\mu}(t)=\tilde{\sigma}_{\mu}(t) e^{i \omega t}, \quad \epsilon(t)=\tilde{\epsilon}(t) e^{i \omega t}, \quad \epsilon^{0}(t)=\hat{\epsilon}^{0}(t) e^{i \omega t}$,

$\sigma_{i j}^{D}(t)=\hat{\sigma}_{i j}^{D}(t) e^{i \omega t}, \quad \epsilon_{i j}^{D}(t)=\bar{\epsilon}_{i j}^{D} e^{i \omega t}$

Substituting these expressions and the corresponding time rates, e.g., $\dot{\sigma}_{\mu}=\left(\dot{\tilde{\sigma}}_{\mu}+i \omega \tilde{\sigma}_{\mu}\right) e^{i \omega t}$ into Eq. 3 for the volumetric components, we obtain

$\frac{\dot{\tilde{\sigma}}_{\mu}}{3 K_{\mu}(t)}+\tilde{\sigma}_{\mu}\left(\frac{1}{3 \eta_{\mu}(t)}+\frac{i \omega}{3 K_{\mu}(t)}\right)=3 K_{\mu}(t)\left[\dot{\tilde{\epsilon}}-\dot{\hat{\epsilon}}^{0}+i \omega\left(\tilde{\boldsymbol{\epsilon}}-\tilde{\epsilon}^{0}\right)\right] \ldots \ldots$

or $\dot{\bar{\sigma}}_{\mu}+\frac{1}{\beta_{\mu}} \tilde{\sigma}_{\mu}=c_{\mu}$

in which we introduce the notations

$\frac{1}{\beta_{\mu}}=\frac{\bar{K}_{\mu}}{\bar{\eta}_{\mu}}+i \omega$,

$c_{\mu}=\frac{3 \tilde{K}_{\mu}}{\Delta t}\left[\Delta \tilde{\boldsymbol{\epsilon}}-\Delta \overline{\boldsymbol{\epsilon}}^{0}+i \omega \Delta t\left(\tilde{\boldsymbol{\epsilon}}_{r}-\tilde{\boldsymbol{\epsilon}}_{r}^{0}+\frac{\Delta \tilde{\boldsymbol{\epsilon}}-\Delta \tilde{\boldsymbol{\epsilon}}^{0}}{2}\right)\right]$

Here again $\Delta$ denotes the increments from $t_{r}$ to $t_{r+1}$

The basic idea in deriving the exponential algorithm for steady loads is to assume that the rates of strain and shrinkage strain, as well as the values of elastic moduli and viscosities of each Maxwell unit, are constant within each time interval, $\Delta t$, while they may change by jumps between the intervals, i.e., at times $t_{r}(r=1,2, \ldots)$. This suggests the use of the same basic idea for the case of fluctuating components. We solve Eq. 14 under the assumption that $c_{\mu}$ and $\beta_{\mu}$ are constant from $t_{r}$ to any time $t \leq t_{r+1}$. Under this assumption, the integral of Eq. 14 for $t_{r} \leq t \leq t_{r+1}$ is

$\tilde{\sigma}_{\mu}(t)=\tilde{\sigma}_{\mu_{r}} \beta_{\mu} c_{\mu} e^{-\left(t-t_{r}\right) / \beta_{\mu}}+\beta_{\mu} c_{\mu}$

in which $\beta_{\mu}$ may be interpreted as the effective volumetric relaxation time for the $\mu$ th Maxwell unit. Note that $\beta_{\mu}$ and $c_{\mu}$ are complex. Substituting in Eq. $16 t=t_{r+1}=t_{r}+\Delta t$, we obtain the values of the complexvalued volumetric partial stresses at the next discrete time as

$\tilde{\boldsymbol{\sigma}}_{\mu, r+1}=\tilde{\boldsymbol{\sigma}}_{\mu, r} e^{-\Delta y_{\mu}}+\kappa_{\mu} c_{\mu} \Delta t$

in which $\Delta y_{\mu}=\frac{\Delta t}{\beta_{\mu}}, \quad \kappa_{\mu}=\frac{1-e^{-\Delta y_{\mu}}}{\Delta y_{\mu}}$

Substituting Eq. 17 into the relation $\Delta \tilde{\sigma}=\Sigma_{\mu}\left(\tilde{\sigma}_{\mu, r+1}-\tilde{\sigma}_{\mu, r}\right)$, we obtain $\Delta \tilde{\sigma}=3 K^{\prime \prime} \Delta \bar{\epsilon}-\Delta \sigma^{\prime \prime}$

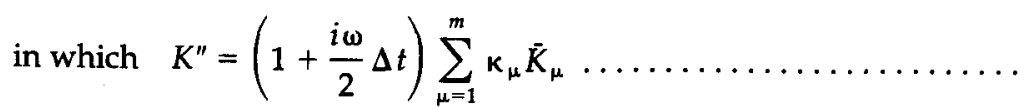

$\Delta \sigma^{\prime \prime}=\sum_{\mu=1}^{m}\left(1-e^{-\Delta y_{\mu}}\right) \tilde{\boldsymbol{\sigma}}_{\mu, r}+3 K^{\prime \prime}\left[\Delta \tilde{\boldsymbol{\epsilon}}^{0}-\frac{i \omega \Delta t}{1+\frac{i \omega}{2} \Delta t}\left(\tilde{\boldsymbol{\epsilon}}_{r}-\tilde{\boldsymbol{\epsilon}}_{r}^{0}\right)\right]$

Obviously, Eq. 19 may be regarded as an incremental volumetric elastic stress-strain relation with a complex-valued incremental bulk modulus, $K^{\prime \prime}$, the value of which is different in each time step; $\Delta \sigma^{\prime \prime}$ represents the complex-valued inelastic volumetric stress increments, which lead, in finite element analysis, to equivalent inelastic nodal force increments (complex-valued) obtained (according to the principle of virtual work) by integration over the element volume.

It should be noted that if $\Delta t / \beta_{\mu}$ is very large, then $\Delta y_{\mu}$ is also very large and $e^{-\Delta y_{\mu}} \rightarrow 0$ and $\lambda_{\mu} \rightarrow 0$. This causes, in similarity to the algorithm for nonfluctuating components, those units whose relaxation times are much shorter than $\Delta t$ to behave as if uncoupled, i.e., they present no resistance to deformation, which is why long time steps are possible with this algorithm. On the other hand, if $\Delta t / \beta_{\mu}$ is very small, then $\Delta y_{\mu}$ is also very small, and $\exp \left(-\Delta y_{\mu}\right) \rightarrow 1$ and $\kappa_{\mu} \rightarrow 1$. This means that those Maxwell units for which the relaxation time is much longer than the time step behave essentially as elastic springs as may be intuitively expected. Note that, in order to avoid an overflow, the value of exp $\left(-\Delta y_{\mu}\right)$ must be programmed as $1-\Delta y_{\mu}+(1 / 2) \Delta y_{\mu}^{2}$ and $\kappa_{\mu}$ as $1-(1 /$ 2) $\Delta y_{\mu}+(1 / 6) \Delta y_{\mu}^{2}$ when $\Delta y_{\mu}$ is too small, and as 0 when $\Delta y_{\mu}$ is too large.

An entirely analogous derivation for the deviatoric components yields, instead of Eq. 17, the following equation:

$\tilde{\sigma}_{i j \mu, r+1}^{D}=\bar{\sigma}_{i j_{\mu, r}}^{D} e^{-\Delta y_{\mu}^{\prime}}+\kappa_{\mu}^{\prime} c_{\mu}^{\prime} \Delta t \ldots$

in which $\Delta y_{\mu}^{\prime}=\frac{\Delta t}{\beta_{\mu}^{\prime}}, \quad \kappa_{\mu}^{\prime}=\frac{1-e^{-\Delta y_{\mu}^{\prime}}}{\Delta y_{\mu}^{\prime}}$

$\frac{1}{\beta_{\mu}^{\prime}}=\frac{\bar{G}_{\mu}}{\bar{\eta}_{\mu}^{D}}+i \omega, \quad c_{\mu}^{\prime} \Delta t=2 \bar{G}_{\mu}\left[\Delta \tilde{\epsilon}_{i j}^{D}+i \omega \Delta t\left(\bar{\epsilon}_{i j_{r}}^{D}+\frac{1}{2} \Delta \bar{\epsilon}_{i j}^{D}\right)\right] \ldots$

This then leads to the deviatoric incremental stress-strain relation

$\Delta \tilde{\sigma}_{i j}^{D}=2 G^{\prime \prime} \Delta \tilde{\epsilon}_{i j}^{D}-\Delta \sigma_{i j}^{D \prime}$

in which $G^{\prime \prime}=\left(1+\frac{i \omega}{2} \Delta t\right) \sum_{\mu=1}^{m} \kappa_{\mu}^{\prime} \bar{G}_{\mu} \ldots \ldots \ldots \ldots \ldots \ldots \ldots \ldots$

$\Delta \sigma_{i j}^{D^{\prime \prime}}=\sum_{\mu=1}^{m}\left(1-e^{-\Delta y_{\mu}^{\prime}}\right) \tilde{\sigma}_{i j_{\mu, r}}^{D}-2 G^{\prime \prime} \frac{i \omega \Delta t}{1+\frac{i \omega}{2} \Delta t} \bar{\epsilon}_{i j r}^{D}$

$G^{\prime \prime}=$ the complex-valued incremental shear modulus, different for each time step; and Eq. 27 gives the deviatoric inelastic stress relaxations (complex-valued) which have to be integrated over the element volume to yield the complex valued increments of the inelastic nodal forces in finite element analysis.

The well-known exponential algorithm for steady, non-periodic, loads 
must be obtained as a special case of Eqs. 19-27 for $\omega \rightarrow 0$. One may easily check that these equations indeed reduce to Eqs. 6-11.

\section{Complex Exponential Algorithm}

For periodically varying stresses and strains (Eq. 12), an algorithm based on Eqs. 19-27 would allow arbitrary increase of the time step. There is a problem, however. Due to aging as well as the initial conditions, the solution of the periodic components cannot be separated from the solution of the drifting mean. How can this be overcome?

One must exploit the fact that the usual exponential algorithm for nonperiodic loads (Eqs. 6-11) yields a nonperiodic response, representing the mean response, when the time-steps $\Delta t$ become much larger than the fluctuation periods. Thus, the equations of this algorithm may be used to isolate the drifting mean component. The remainder, then, is the periodic component of response to which Eqs. 19-27 are applicable. Therefore, in a step-by-step algorithm, it is possible to apply in each time step first Eqs. 6-11 to determine the increments of the drifting mean response, and subsequently apply Eqs. 19-27 to determine the changes in the (complex-valued) amplitudes of the remaining periodic components of the response. In each time step, the following algorithm, in which the distinction between the drifting mean variables and the superimposed periodic variables is provided by the fact that the first ones are real and the second ones are complex, may be used.

First solve the increments of real variables:

1. Evaluate Eqs. $10,9,7,8$.

2. Consider the change, $\hat{h}\left(t_{r+1}\right)-\hat{h}\left(t_{r}\right)$, of the mean environmental humidity (or temperature) from $t_{r}$ to $t_{r+1}$ as the prescribed changes in boundary conditions (input, loading), then calculate the increments in the mean (real-valued) shrinkage $\Delta \hat{\boldsymbol{\epsilon}}^{0}$ from $t_{r}$ to $t_{r+1}$, and then evaluate the real-valued inelastic stress changes from $\mathrm{Eq} .8$.

3. For these increments of loads and shrinkage (or thermal strain), solve the real-valued stress and strain changes, $\Delta \hat{\sigma}$ and $\Delta \hat{\epsilon}$, by elastic finite element analysis based on Eq. 6 .

4. Solve the new values of the real-valued hidden stresses from Eq. 10 (for all finite elements and all integration points in them).

Second, solve the complex-valued stress and strain changes for the same time step:

5. Evaluate Eqs. 18, 23, 20, and 26.

6 . Consider the complex-valued changes in the amplitude of the periodic component of environmental humidity (or temperature), $\Delta \tilde{h}$, from $t_{r}$ to $t_{r+1}$, and calculate the corresponding complex-valued increments of the amplitude of the periodic shrinkage component, $\Delta \tilde{\epsilon}^{0}$, from $t_{r}$ to $t_{r+1}$

7. For these complex-valued changes of shrinkage (input, loading) from $t_{r}$ to $t_{r+1}$, solve by finite elements the incremental elastic problem based on the stress-strain relations in Eqs. 19 and 25. This yields the increments in the complex-valued stresses and strains.

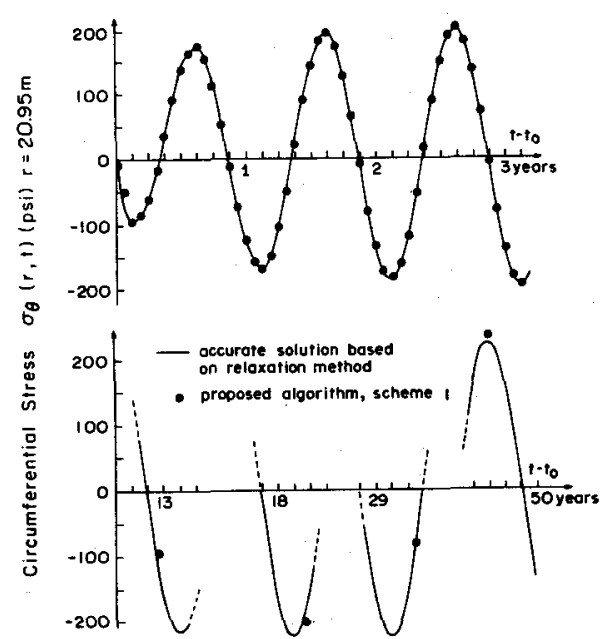

FIG. 1.-Comparison of Solutions Obtalned by Different Methods

8. Solve the new values of the complex-valued partial stresses from Eqs. 22 and 27 (for all finite elements).

9. Adding the increments of the real-valued and complex-valued stresses and strains, obtain the values for the end of the step, $t_{r+1}$, and advance to the next time step by returning to item 1 , unless the final time has been reached.

To start the calculation, the first time step, $\Delta t$, must be chosen to be much smaller than the fluctuation period, $T=2 \pi / \omega$. At the beginning of the calculation, when the time-step, $\Delta t$, is still small and much shorter than the fluctuation period of the input (environment), the real part of the solution can, itself, accurately follow the fluctuations, and the complex-valued response is then obtained as 0 . The complex-valued response becomes nonzero as the time step approaches the value of the fluctuating period. The real part of the response does not represent the drifting mean of the response until the time step $\Delta t$ becomes much longer than the oscillation period. Thus, the interpretation of the response parts as the fluctuating and drifting mean parts, as introduced in Eqs. 4-5, applies only during the later stage of calculation when the time step is much larger than the periods. During the initial stage of the calculations, Eqs. 4-5 cannot be literally interpreted as a separation of fluctuating and drifting mean components, but that causes no problem since the total response $\sigma_{i j}(t)$ and $\epsilon_{i j}(t)$ is correct.

Note that the time step $\Delta t$ does not have to be an integer multiple of the fluctuation period, $T$. The discrete times may fall onto the fluctuating response curve at any point, as exemplified in Fig. 1 . Thus, one discrete time may fall onto the peak of response, the next one onto the trough, the next one onto the mean, etc.

The same as for the standard exponential algorithm for steady loads, the time steps, $\Delta t$, may be chosen to be constant in the scale of log 
$\left(t-t_{0}\right)$. The time steps then form a geometric progression. As mentioned, the first $\Delta t$ must be much less than the shortest fluctuation period, $T=2 \pi / \omega$.

\section{EXAMPLES}

Example 1.-We choose the same example as in Ref. 10 and solve a hollow concrete cylinder of infinite length, whose inner and outer radii are $20 \mathrm{~m}$ and $21 \mathrm{~m}$, respectively. The drying diffusivity of concrete is constant, $C_{0}=0.3 \mathrm{~cm}^{2} /$ day. The Poisson ratio is $\nu=0.18$. The environmental relative humidity varies sinusoidally with the period $T=365$ days, $h=h_{m}+A \cos \omega\left(t-t_{0}\right)=0.7+0.2 \cos [2 \pi(t-28) / 365]$, i.e., its mean annual value is $h_{m}=0.7$, its amplitude is $A=0.2$, and its circular frequency is $\omega=2 \pi / 365$. The age of concrete at the beginning of exposure to the environment is $t_{0}=28$ days. In order to separate the effect of the difference between the initial pore humidity, $h_{0}$, and the mean environmental humidity, $h_{m}$, which does not cause oscillating response, we choose $h_{0}=h_{m}$, i.e., $h_{0}=0.7$, for our illustrative example. The shrinkage strains are assumed to be proportional to the changes of pore humidity, with proportionality coefficient (shrinkage coefficient) $\kappa_{\text {sh }}$ $=0.0008$.

Aside from the stress and strain analysis described in the foregoing, the solution also requires solving the diffusion problem of pore humidity, $h$. The calculation has been carried out in the same manner as described in Ref. 10, and it yields the numerical values for the following function:

$h(r, t)=H\left(\omega, r, t, t_{0}\right) e^{i \omega t}$

in which $H$ denotes the frequency response function of pore humidity; and $r=$ the radius coordinate. Both $h$ and $H$ are complex-valued. Multiplying Eq. 28 by $\kappa_{s h}$ yields the values of the shrinkage strains which represent the loading terms for the stress and strain analysis and figure in the algorithm described earlier. Because we chose $h_{m}=h_{0}$, only the complex-valued terms in Eqs. 4 and 5 need to be dealt with.

In each time step, the elastic stress and strain analysis is carried out using one-dimensional finite elements (rings) obtained by subdividing the radius as shown in Fig. 2. The number of elements is 17; 7 elements of $10 \mathrm{~cm}, 5$ elements of $4 \mathrm{~cm}$, and 5 elements of $2 \mathrm{~cm}$. The time step subdivision schemes that have been used are shown in Fig. 3 and Table 1.
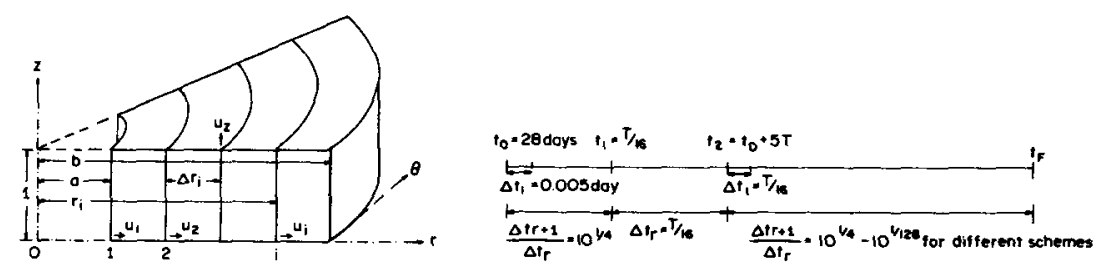

FIG. 2.-One-Dimensional Discretlza- $\begin{aligned} & \text { FIG. 3.-TIme Step Subdivision } \\ & \text { tion of Cylindrical Wall }\end{aligned}$
Schemes
TABLE 1-Time Step Subdivision Schemes

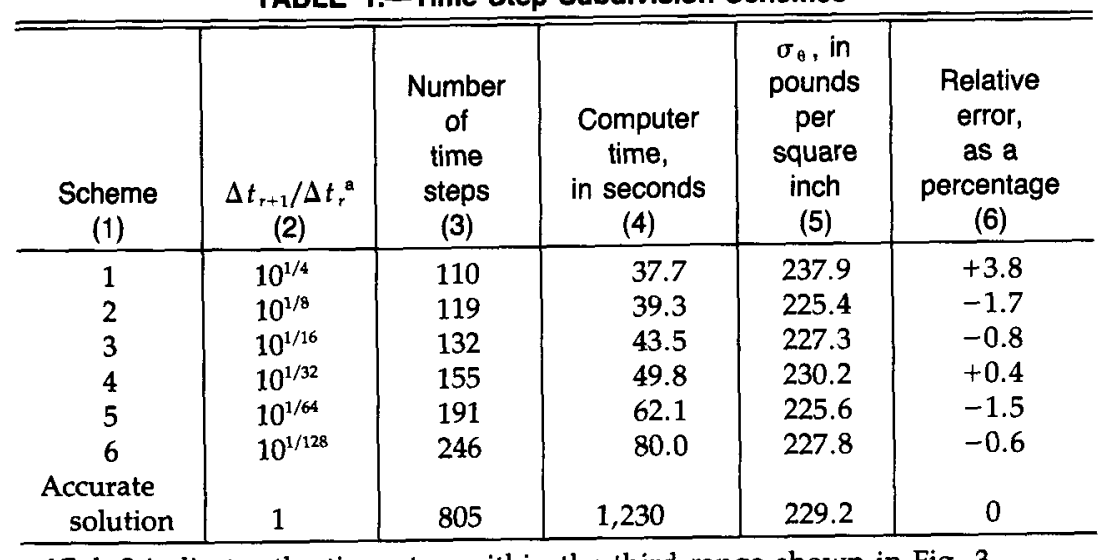

${ }^{a}$ Col. 2 indicates the time step within the third range shown in Fig. 3

Note: $\sigma_{\theta}=$ peak value at $r=20.95 \mathrm{~m}$ and at $t_{F}=18,141$ days $\approx 50 \mathrm{yr}$; accurate solution based on relaxation function (Ref. 2 ); $T=365$ days; $\Delta t_{r}=T / 16=22.81$ days.

Because $a>b-a$, the values of $\sigma_{\theta}$ and $\sigma_{z}$ are so close that they cannot be distinguished. Due to the same reason, the value of $\sigma_{r}$ is very low and insignificant. Therefore, only $\sigma_{\theta}$ is given in the following figures and tables.

The solution obtained is exemplified by the plots in Fig. 1, in which the locations of discrete times are shown by dots. The figure shows portions of the calculated history of the circumferential normal stress at depths $5 \mathrm{~cm}$ below the outer surface.

A comparison of the present solution with that obtained previously (10), using very short time steps, is given in Fig. 1 and Table 1. For scheme 1 the errors seem to be generally within $4 \%$. Although the present solution does not converge to the exact one monotonically, an improvement can be achieved with a finer, but still increasing, time subdivision. For a life span of $50 \mathrm{yr}$, the computer time (using CDC/Cyber)

TABLE 2.-SInusoidal Environmental Humidity Solution

\begin{tabular}{c|c|c|c|c}
\hline \hline $\begin{array}{c}\text { Scheme } \\
(1)\end{array}$ & $\begin{array}{c}\Delta t_{r+1} / \Delta t_{r}{ }^{a} \\
(2)\end{array}$ & $\begin{array}{c}\text { Number of } \\
\text { time steps } \\
(3)\end{array}$ & $\begin{array}{c}\text { Computer time, } \\
\text { in seconds } \\
(4)\end{array}$ & $\begin{array}{c}\sigma_{\theta}, \text { in } \\
\text { pounds per } \\
\text { square inch } \\
(5)\end{array}$ \\
\hline 1 & $10^{1 / 4}$ & 110 & 37.7 & 217.6 \\
2 & $10^{1 / 8}$ & 122 & 40.6 & 225.9 \\
3 & $10^{1 / 16}$ & 150 & 47.9 & 195.1 \\
4 & $10^{1 / 32}$ & 196 & 62.6 & 215.4 \\
5 & $10^{1 / 64}$ & 278 & 88.8 & 198.4 \\
6 & $10^{1 / 128}$ & 423 & 135 & 205.3 \\
\hline
\end{tabular}

${ }^{a}$ Col. 2 indicates the time step within the third range shown in Fig. 3.

Note: $\sigma_{\theta}=$ peak value at $r=20.99 \mathrm{~m}$ and at $t_{F}=18,276$ days $\simeq 50 \mathrm{yr} ; T=14$ days; $\Delta t_{r}=T / 16=0.88$ days. 


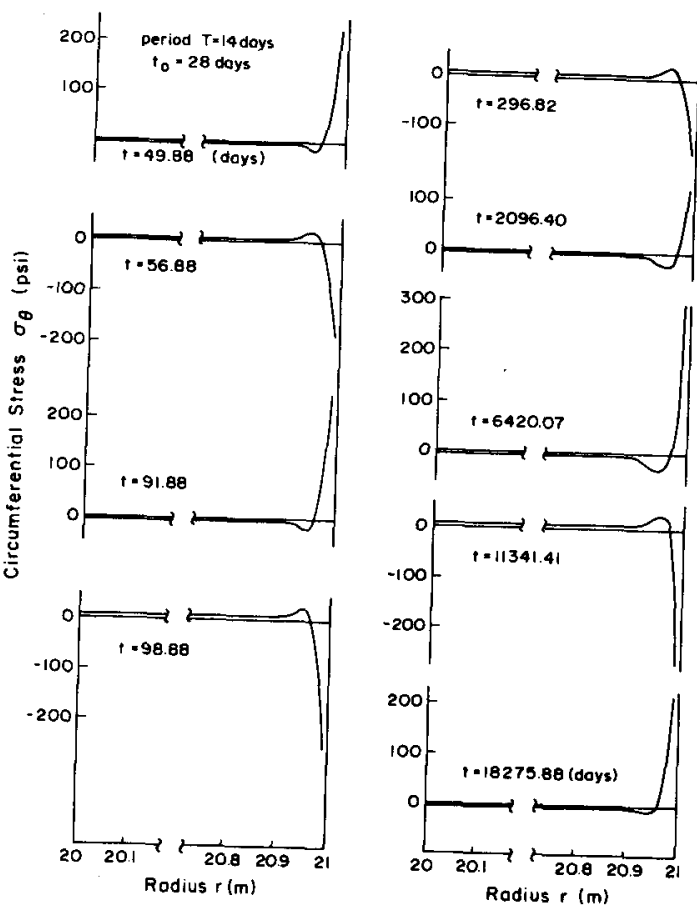

FIG. 4.-Distribution of Circumferential Shrinkage Stress In Cylindrical Wall

was about 33 times shorter for the present solution of scheme 1 , compared to that in Ref. 10. A saving of this magnitude would be essential finite element system.

Example 2.-For a second example, we chose a sinusoidal environmental humidity with a very short period, $T=14$ days, i.e., $h=0.55$ $+0.2 \cos [2 \pi(t-28) / 14]$. All other given data for this example are the same. The solution for this example is given in Table 2, and the distributions of stresses are shown in Fig. 4. The time division is defined in Fig. 3 and Table 2. Although the solution does not division is defined in one monotonically, one may assume that the ave converge to the exact of schemes 3-6 could be considered the average of the solutions ratio of $\Delta t+1$ could be considered as the correct solution. Then, if the improvement $\Delta t_{r}$ is chosen $1 / 16$, the errors appear to be within $6 \%$. An improvement can again be achieved with a finite, but still increasing, time subdivision. It may be estimated that the previously reported solake with time steps much smaller than the fluctuation periods would take about 600 times more computer time than the present solution. This
increase in efficiency is remarkable.

It may be noted that, for the shorter environmental fluctuation period, expected (approximately up to $5 \mathrm{~cm}$ beneath the outer surface) (Fig. 4).
(F)

\section{Conclusions}

1. Assuming that the material properties and the strain rates are con- stant within each time step, and using an exact integral of the rate-type stress-strain relations, one may develop, for strictly periodic response, a complex exponential algorithm which is completely analogous to the well-known exponential algorithm for steady loading, the only difference being that all variables are complex rather than real.

2. Since the periodic and drifting mean parts of the response cannot be separated in advance, due to aging and the initial condition, the exponential algorithm must be combined and run simultaneously with the standard exponential algorithm. This approach allows increasing the time step in a geometric progression, ending with time steps that are orders of magnitude larger than the prescribed fluctuation period.

3. This new complex exponential algorithm allows a significant saving of computer time, especially when many cycles of short periods are to be solved.

4. The new algorithm is useful mainly for spectral analysis of creep and shrinkage effects in concrete structures subjected to a random environment of a given spectral density.

\section{ACKNOWLEDGMENT}

Thanks are due to the U.S. National Science Foundation for partially supporting this research under Grant No. CEE-8303148. The outstanding secretarial assistance of Mary Hill deserves mention.

\section{ApPendix.-References}

1. ASCE Structural Division Task Committee on Finite Element Analysis of Reinforced Concrete (chaired by A. Nilson), "Finite Element Analysis of Reinforced Concrete (State-of-the-Art Report)," ASCE, New York, N.Y., 1982, Chpt. 6, pp. 309-400.

2. Bažant, Z. P., "Numerical Analysis of Long-Time Deformations of a ThickWalled Concrete Cylinder," Report No. 69-12, Structure and Materials Research, Civil Engineering Department, University of California, Berkeley, Calif., Aug., 1969.

3. Bažant, Z. P., "Numerically Stable Algorithm With Increasing Time Steps for Integral-Type Aging Creep," 1st International Conference on Structural Mechanics in React. Tech., Berlin, Vol. 4, Paper H2/3, Sept., 1971.

4. Bazant, Z. P. "Theory of Creep and Shrinkage in Concrete Structures: A Précis of Recent Developments," Mechanics Today, Vol. 2, 1-93, Pergamon Press, New York, N.Y., 1975.

5. Bažant, Z. P., "Mathematical Models for Creep and Shrinkage of Concrete," Chpt. 7, Creep and Shrinkage in Concrete Structures, Z. P. Bažant and F. H. Wittmann, eds., John Wiley, London, 1982.

6. Bažant, Z. P., "Response of Aging Linear Systems to Random Input," Concrete and Geomaterials Report No. 82-12/665r, Northwestern University, Evanston, Ill., Dec., 1982.

7. Bažant, Z. P. "Probabilistic Problems in Prediction of Creep and Shrinkage Effects in Structures," Proc. 4th Int. Conf. on Appl. of Statistics and Probability in Soil and Structural Engineering, G. Augusti, ed., Florence, Italy, June, 1983, pp. 325-356.

8. Bažant, Z. P., and Asghari, A., "Computation of Age-Dependent Relaxation Spectra," Cement and Concrete Research, Vol. 4, 1974, pp. 567-579.

9. Bažant, Z. P., and Wang, T. S., "Spectral Analysis of Random Shrinkage Stresses in Concrete Structures," Journal of Engineering Mechanics, ASCE, Vol. 110, No. 2, Feb., 1984, pp. 173-186. 
10. Bažant, Z. P., and Wang, T. S., "Spectral Finite Element Analysis of Random Shrinkage in Concrete Structures," Journal of Structural Engineering, ASCE, to be published.

11. Bažant, Z. P., and Wu, S. T., "Rate-Type Creep Law of Aging Concrete Based on Maxwell Chain," Materials and Structures, Vol. 7, No. 37, Paris, France, 1974, pp. 45-60.

12. Cox, H. J., and Armington, J. H., The Weather and Climate of Chicago, The University of Chicago Press, Chicago, IIl., 1914.

13. Crandall, S. H., Random Vibration in Mechanical Systems, Academic Press, New York, N.Y., 1963.

14. Davenport, W. B., Probability and Random Processes, McGraw-Hill, New York, N.Y., 1970 .

15. Fuller, W., An Introduction to Probability Theory with Applications, 2nd ed., Vol. 2, Chapt. 19, John Wiley \& Sons, New York, N.Y., 1971

16. Neville, A. M., Properties of Concrete, John Wiley, New York, N.Y., 1963.

17. Neville, A. M., and Dilger, W., Creep of Concrete: Plain, Reinforced Prestressed, North Holland Publ. Co., Amsterdam, 1970.

18. Newland, D. E., An Introduction to Random Vibration and Spectral Analysis, McGraw Hill, New York, N.Y., 1975.

19. Papoulis, A., Probability, Random Variables and Stochastic Processes, McGrawHill, New York, N.Y., 1965.

20. Taylor, R. L., Pister, K. S., and Goudreau, G. L., "Thermo-mechanical Analysis of Viscoelastic Solids," International Journal for Numerical Method in Engineering, Vol. 2, 1970, pp. 45-60.

21. Tsubaki, T., and Bažant, Z. P., "Random Shrinkage Stresses in Aging Viscoelastic Vessel," Journal of the Engineering Mechanics Division, ASCE, Vol. 108 No. EM3, June, 1982, pp. 527-545.

22. U.S. Environmental Data Service, "Weather Atlas of the United States," Gale Research Co., Detroit, Mich., 1975

23. Zienkiewicz, O. C., The Finite Element Method, 3rd ed., McGraw-Hill, New York, N.Y., 1977.

24. Zienkiewicz, O. C., Watson, M., and King, I. P. "A Numerical Method of Viscoelastic Stress Analysis," Intern. J. Mech. Sciences, Vol. 10, 1968, pp. 807827. 